\title{
Evaluation of electromyographic activity and heart rate responses to isometric exercise. The role played by muscular mass and type
}

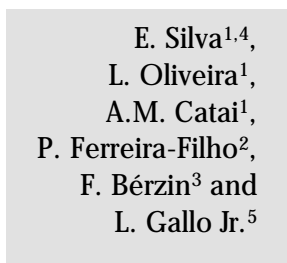

\section{Correspondence}

\section{E. Silva}

Laboratório de Fisioterapia

Cardiovascular

Departamento de Fisioterapia,

UFSCar

Via Washington Luiz, km 235

13565-905 São Carlos, SP

Brasil

Fax: + 55-16-271-2081

E-mail: esters@ power.ufscar.br

Research supported by CN Pq (No. 520.686/95-0). Publication supported by FAPESP.

Received July 20, 1998 Accepted O ctober 28, 1998

\author{
${ }^{1}$ Laboratório de Fisioterapia Cardiovascular, Departamento de Fisioterapia, and \\ ${ }^{2}$ Departamento de Estatística, Universidade Federal de São Carlos, \\ São Carlos, SP, Brasil \\ ${ }^{3}$ Departamento de Morfologia, Faculdade de O dontologia de Piracicaba, \\ Universidade Estadual de Campinas, Piracicaba, SP, Brasil \\ ${ }^{4}$ Departamento de Fisiologia e Biofísica, Universidade Estadual de Campinas, \\ Campinas, SP, Brasil \\ ${ }^{5}$ Divisão de Cardiologia, Hospital das Clínicas, Faculdade de Medicina de Ribeirão \\ Preto, Universidade de São Paulo, Ribeirão Preto, SP, Brasil
}

\section{Abstract}

The purpose of the present study was to examine the relationship between the electromyographic (EMG) activity and heart rate (HR) responses induced by isometric exercise performed by knee extension $(\mathrm{KE})$ and flexion $(\mathrm{KF})$ in men. Fifteen healthy male subjects, $21 \pm 1.3$ years (mean $\pm \mathrm{SD}$ ), were submitted to $\mathrm{KE}$ and $\mathrm{KF}$ isometric exercise tests at $100 \%$ of maximal voluntary contraction (MVC). The exercises were performed with one leg (right or left) and with two legs simultaneously, for $10 \mathrm{~s}$ in the sitting position with the hip and knee flexed at $90^{\circ}$. EMG activity (root mean square values) and HR (beats $/ \mathrm{min}$ ) were recorded simultaneously both at rest and throughout the sustained contraction. The HR responses to isometric exercise in KE and KF were similar when performed with one and two legs. However, the HR increase was always significantly higher in $\mathrm{KE}$ than $\mathrm{KF}(\mathrm{P}<0.05)$, whereas the EMG activity was higher in $\mathrm{KE}$ than in $\mathrm{KF}(\mathrm{P}<0.05)$, regardless of the muscle mass (one or two legs) involved in the effort. The correlation coefficients between HR response and the EMG activity during $\mathrm{KE}(\mathrm{r}=0.33, \mathrm{P}>0.05)$ and $\mathrm{KF}(\mathrm{r}=0.15, \mathrm{P}>0.05)$ contractions were not significant. These results suggest that the predominant mechanism responsible for the larger increase in HR response to KE as compared to KF in our study could be dependent on qualitative and quantitative differences in the fiber type composition found in each muscle group. This mechanism seems to demand a higher activation of motor units with a corresponding increase in central command to the cardiovascular centers that modulate HR control.

\section{Key words}

- Knee

- Isometric exercise

- Heart rate

- Electromyography

- Rectus femoris

- Biceps femoris 
The autonomic efferent mechanisms responsible for the tachycardia induced by isometric exercise are well known. Both vagal withdrawal and a sympathetic stimulation of the sinus node may contribute in different degrees to the exercise tachycardia, depending on the duration and intensity of isometric effort $(1,2)$. However, studies on cardiovascular responses related to isometric exercise involving muscles of different group types show conflicting results. There are reports showing that the increase in heart rate (HR) during isometric exercise is determined by the duration, percentage of maximal voluntary contraction (\%MVC), muscle length, and the amount and size of active muscle mass (3-5). In contrast, some investigators $(6,7)$ have reported that HR response to isometric exercise is related to the intensity of the maximum tension achieved rather than to the amount or size of the mass of the contracting muscle group. Also, it is worth mentioning the findings of other investigators showing that the HR increase is independent of muscle length and the external torque under conditions of controlled relative intensity of the MVC or muscle mass (8). In this context, it should be taken into consideration that muscle length is modified by the joint angle, and the maximal internal muscle force is directly related to muscle length (9). The purpose of the present study was to examine the relationship between the electromyographic (EMG) activity and HR response to isometric exercise in men, performed by a small (one leg) and large (two legs) muscle mass of different muscle groups at the same joint angle, with the same intensity (100\% MVC), for $10 \mathrm{~s}$.

Fifteen healthy male subjects were studied. Their anthropometric characteristics reported as mean and standard deviations were: age, $21 \pm 1.3$ years, weight, $81 \pm 6.2 \mathrm{~kg}$, and height, $182 \pm 3.9 \mathrm{~cm}$. All subjects were submitted to a medical examination prior to the study, including a resting electrocardiogram (ECG). They were informed about the study design and its objectives, and each subject signed a formal consent form, as required by the Ethics Committee of the Universidade Estadual de Campinas. The experimental protocol consisted of isometric exercises of knee extension (KE) and flexion (KF) at $100 \%$ MVC. The exercises were performed using a special chair (10), with the subject in a seated position, with hip and knees in flexion at $90^{\circ}$. The subjects were stabilized in the chair with Velcro straps placed on the chest, pelvis and midthigh, to minimize the contraction of other muscles not directly involved in the exercise test. The resistance pad at the end of the lever arm was strapped approximately $2.5 \mathrm{~cm}$ above the medial malleolus. The subjects were instructed to breathe spontaneously and not to perform a Valsalva maneuver while straining, to avoid reflex HR responses due to cardiovascular changes. They performed six tests of isometric exercises in $\mathrm{KE}$ and $\mathrm{KF}$, with one (left and right leg in random order) and with both legs at $100 \%$ MVC for $10 \mathrm{~s}$. The subjects were verbally instructed by the investigators to use their maximal force and were vigorously encouraged to maintain it at a constant level for a period of $10 \mathrm{~s}$, "Are you ready? Now push! Push harder! Harder! And relax". The interval between two successive tests was set at 10 min when HR values had always returned to basal conditions. The HR was obtained from an ECG. The signals were recorded in real time after analog to digital conversion (ADC), and the identification of "R" peaks of the ECG waves; the R-R intervals were calculated on a beat-to-beat basis using a special software developed in our laboratory (11). Recordings were obtained for $65 \mathrm{~s}$ before each exercise, throughout the sustained contraction, and for $285 \mathrm{~s}$ during the recovery period. The resting HR values (beats/min) correspond to an average obtained during a period of $65 \mathrm{~s}$ immediately before the beginning of exercise and the peak HR response was expressed as the highest instantaneous value attained at $10 \mathrm{~s}$ of 
exercise $(\Delta \mathrm{HR}=0-10 \mathrm{~s})$. The HR response corresponding to the effort of the right or left leg during the isometric exercise with only one leg was expressed as the mean value of the two legs.

EMG activity was recorded using a multichannel electromyographic system (Nicolet Viking II) with a self-adhesive Beckman surface mini-bipolar electrode, consisting of two silver/silver chloride electrodes and a solid-state preamplifier in a plastic enclosure, applied to the skin over the following muscle groups: 1) right and left rectus femoris; 2) right and left biceps femoris; 3 ) right and left intercostal muscles between the 5 th and 6th intercostal spaces, and left pectoralis major. The rectus femoris and biceps femoris muscle groups were mainly involved in knee extension and flexion, respectively.

EMG signals were also recorded in real time after ADC, sampled at a frequency of $250 \mathrm{~Hz}$, with band filters having a low cutoff frequency and a high cut-off frequency set at $10 \mathrm{~Hz}$ and $10 \mathrm{kHz}$, respectively. Using the MMP software (Multi Mode Program) the data were processed and presented as graphic and numerical values. The EMG data included the $10 \mathrm{~s}$ of muscular effort $(100 \% \mathrm{MVC})$ as well as $5 \mathrm{~s}$ before and after each test. The magnitude of the EMG signal in microvolts $(\mu \mathrm{V})$ was presented as the root mean square values (RMS) for the purposes of quantifying the electromyographic activity during each isometric contraction. The EMG signal corresponding to the effort of the right or left leg during the isometric exercises with only one limb is reported as the mean value of the two legs.

The results are presented in a box-plot as median, 1 st and 3rd quartiles, range and outliers. Statistical analysis was conducted using the Friedman nonparametric multiple comparison tests. The relationship between the EMG and HR responses to exercise was reported using Spearman rank correlation coefficients (12). The differences were considered significant at the $\mathrm{P}<0.05$ level. Analy- sis was performed using the software package STATISTICA for Windows, Release 5.1. StatSoft, Inc. 1994-1996 and BMDP 3S Nonparametric Statistics software package.

HR values at rest were always similar immediately before the knee extension and flexion isometric tests. The HR increased significantly above the control levels under all exercise conditions $(\mathrm{P}<0.05)$. Peak HR $(\Delta \mathrm{HR}=0-10 \mathrm{~s})$ attained at the end of the isometric exercise was significantly higher in $\mathrm{KE}$ than $\mathrm{KF}(\mathrm{P}<0.05$; Figure 1). However, the magnitude of the HR increment induced by isometric exercise for each modality of contraction (KE and KF) was similar when exercise was performed with one and two legs $(\mathrm{P}>0.05$, Figure 1$)$

The EMG signal $(\mu \mathrm{V})$ values (RMS) during each trial of sustained contractions were always higher in $\mathrm{KE}$ than $\mathrm{KF}$, independently of whether contraction was performed with one or two legs $(\mathrm{P}<0.05$; Figure 2$)$. The correlation between the RMS values of the EMG signal of the contracting muscles and the HR responses was not statistically significant for $\mathrm{KE}(\mathrm{r}=0.33, \mathrm{P}>0.05)$ or $\mathrm{KF}$ $(r=0.15, P>0.05)$.

Considering the time course and the magnitude of the HR response to maximal isometric exercise at $10 \mathrm{~s}$, our results under all conditions were similar to those reported in the literature $(1,2)$. However, our results show that the quantity of muscle mass involved did not appear to have a significant influence on the HR response under the conditions studied, i.e., maximal isometric contraction with one or two legs (approximately twice the quantity of muscle mass), involving extension or flexion of the knee, with hip and knee positioned at $90^{\circ}$ flexion. Nevertheless, we observed that the magnitude of the HR response was significantly greater in the extension than in the flexion isometric exercise.

The EMG activity and HR responses to the MVC effort found in the present study are in agreement with recent observations 
Figure 1 - Heart rate $(\Delta \mathrm{HR}$ beats/min) responses at $10 \mathrm{~s}$ of maximal isometric exercise tests. (A) and (C) report the values for the unilateral right and left extension and flexion exercises, and (B) and (D) report the values for the bilateral knee extension and flexion exercise. Data are reported as medians, 1 st, and 3rd quartiles and range.

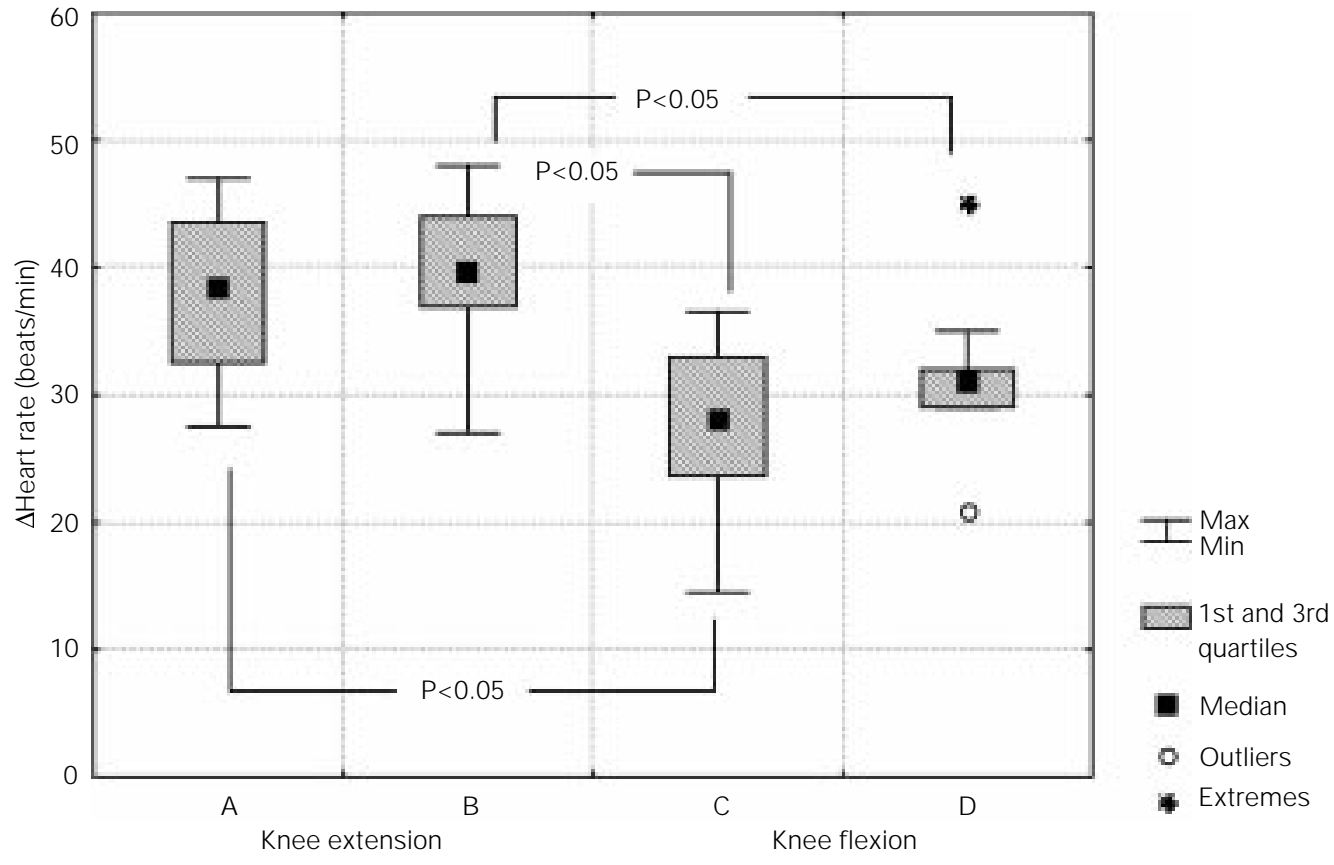

that the magnitude of these variables is influenced by the types of muscles participating in the process of muscle contraction (4-6). In this context, our data support the idea that the different HR responses are related to the activation of the motor units of the muscle groups involved in the contraction process. In relation to these findings, it should be emphasized that the magnitude of the EMG and HR responses could be significantly different as a function of the structural and functional characteristics of a particular muscle fiber found in each muscle group during contraction $(4,13)$, depending on its slow (type I, red, tonic, and aerobic fibers) or fast (type II, white, and anaerobic fibers) nature. It has been found that slow contraction fibers compared to fast contraction fibers present: 1) a low threshold for recruitment of the motor neurons, 2) higher firing rates, 3) a high energetic efficiency and resistance to fatigue, and 4) a lower ratio between frequency and depolarization during the production of force (13). Also, it has been noted that during force generation the motor units with a high threshold for recruitment begin to fire at higher rates than the lower ones, which could cause the occurrence of an early fatigue and as a consequence a greater recruitment of motor units to maintain the initial force $(9,13)$. Another aspect to be considered is that the relative quantity of fast and slow muscle fibers in humans differs in size and number for each specific muscle group and even in the same group depending on their functional specificity. Thus, these findings are in accordance with our present study, since the main muscles involved in knee extension and flexion, the rectus femoris and the biceps femoris, also have a higher percentage of fast and slow twitch fibers, respectively $(14,15)$.

These results suggest that the additional recruitment of motor units for the development and maintenance of the maximal voluntary effort of extension, with the right and/ or left leg, could be attributed to the differences in the percentages of fiber types found in muscles involved in the experimental conditions studied. As a consequence of these arguments, the increase in the recruitment of the motor fibers involved in knee extension should demand greater activation of central command $(3,4)$. 


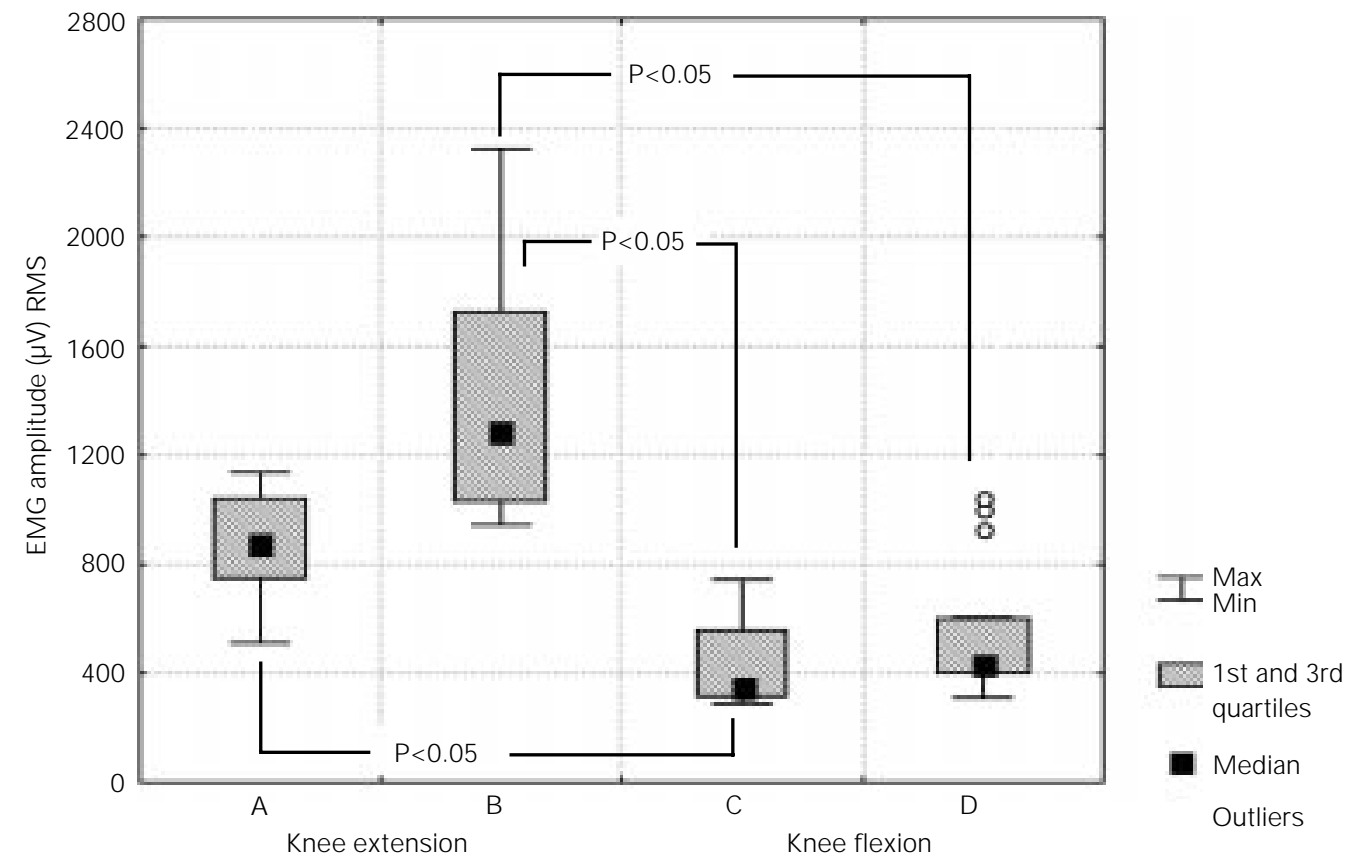

It is possible that the lack of correlation between our EMG and HR responses could be due to a non-linear relationship between these two variables. Under these circumstances it is plausible to propose that the explanation for the absence of an increase in magnitude of the HR response in two-legs versus one-leg conditions might be due to saturation of central irradiation over cardiovascular bulbar centers. Finally, we should take into consideration that the differences between our results and those of other studies $(7,8)$ concerning the relationship of EMG signal and HR may also be related to diversity of experimental protocols, since most of them were conducted on muscle groups of

\section{References}

1. Martin CE, Shaver J A, Leon DF, Thompson ME, Reddy PS \& Leonard J J (1974). Autonomic mechanisms in hemodynamic responses to isometric exercise. J ournal of Clinical Investigation, 54: 104-115.

2. Maciel BC, Gallo J $r$ L, Marin-Neto J A \& Martins LEB (1987). Autonomic nervous control of the heart rate during isometric exercise in normal man. Pflügers Archives, European J ournal of Physiology, different type and size, at levels usually below $100 \%$ MVC and with longer time contraction.

In conclusion, our data support the idea that under conditions of maximum isometric effort the main modulating mechanism of the HR response is related to the specificity of the central command for the muscle groups with their corresponding pattern of fiber recruitment.

\section{Acknowledgments}

The authors are deeply grateful to Vera Regina Fernandes da Silva Marães and Carlos Augusto Lemos Mega for technical assistance.
Figure 2 - Amplitude of electromyographic signals are reported as root mean square values (RMS) $(\mu \mathrm{V})$ at $10 \mathrm{~s}$ of maximal isometric exercise tests, with knee extension and flexion. (A) and (C) report the values for the unilateral right and left extension and flexion exercises and (B) and (D) report the RMS values, as a sum of both knees, from bilateral extension and flexion exercises. Data are reported as medians, 1st and 3rd quartiles and range.
408: 173-177.

3. Ramos UM, Mundale MO, Awad EA, Witsoe DA, Cole TM, Olson M \& Kottke FJ (1973). Cardiovascular effects of spread of excitation during prolonged isometric exercise. Archives of Physical Medicine and Rehabilitation, 54: 496-504.

4. Mitchell J H, Pyne HR, Saltin B \& Schibye $B$ (1980). The role of muscle mass in the cardiovascular response to static contrac- tions. J ournal of Physiology, 309: 45-54.

5. Seals DR, Washburn RA, Hanson PG, Painter PL \& Nagle FJ (1983). Increased cardiovascular response to static contraction of larger muscle groups. J ournal of Applied Physiology: Respiratory Environmental and Exercise Physiology, 54: 434437.

6. McCloskey DI \& Streatfeild KA (1975). Muscular reflex stimuli to the cardiovas- 
cular system during isometric contractions of muscle groups of different mass. J ournal of Physiology, 250: 431-441.

7. Williams CA (1991). Effect of muscle mass on the pressor response in man during isometric contractions. J ournal of Physiology, 435: 573-584.

8. NG AV, Agre J C, Hanson P, Harrington MS \& Nagle FJ (1994). Influence of muscle length and force on endurance and pressor responses to isometric exercise. J ournal of Applied Physiology, 76: 2561-2569.

9. Basmajian JV \& De Luca CJ (1985). Muscle Alive, Their Functions Revealed by Electromyography. 5th edn. Williams
$\&$ Wilkins, Baltimore.

10. Monteiro-Pedro V (1995). Atividade eletromiográfica do músculo vasto medial oblíquo em esforços isométricos e isotônicos das articulações do quadril e do joelho. Doctoral thesis, Faculdade de Odontologia de Piracicaba, UNICAMP, Piracicaba.

11. Silva E, Catai AM, Trevelin LC, Guimarães J O, Silva J r LP, Silva LMP, Oliveira L, Milan LA, Martins LEB \& Gallo J r L (1994). Design of computerized system to evaluate the cardiac function during dynamic exercise. Physics in Medicine and Biology, 33: 409 (Abstract).

12. Siegel S (1956). Nonparametric Statistics for the Behavioral Sciences. McGraw-Hill, Tokyo.

13. Erim Z, De Luca CJ, Mineo $K \&$ Aoki $T$ (1996). Rank-ordered regulation of motor units. Muscle and Nerve, 19: 563-573.

14. Polgar J, J ohnson MA, Weigtman D \& Appleton D (1973). Data on fibre size in thirty-six human muscles - an autopsy study. J ournal of Neurological Sciences, 19: 307-318.

15. J ohnson MA, Weightman $D \&$ Applenton D (1973). Data on the distribution of fibre types in thirty-six human muscles. J ournal of Neurological Sciences, 18: 111-129. 